Claremont Colleges

Scholarship@ Claremont

All HMC Faculty Publications and Research

HMC Faculty Scholarship

8-1-1997

\title{
Quasi-steady Monopole and Tripole Attractors in Relaxing Vortices
}

Louis F. Rossi

University of Massachusetts - Lowell

Joseph F. Lingevitch

Naval Research Laboratory

Andrew J. Bernoff

Harvey Mudd College

\section{Recommended Citation}

Quasi-steady monopole and tripole attractors for relaxing vortices. Louis F. Rossi, Joseph F. Lingevitch, and Andrew J. Bernoff, Phys. Fluids 9, 2329 (1997).

This Article is brought to you for free and open access by the HMC Faculty Scholarship at Scholarship @ Claremont. It has been accepted for inclusion in All HMC Faculty Publications and Research by an authorized administrator of Scholarship @ Claremont. For more information, please contact 


\title{
Quasi-steady monopole and tripole attractors for relaxing vortices
}

\author{
Louis F. Rossi \\ Mathematical Sciences Department, University of Massachusetts, Lowell, Massachusetts 01854 \\ Joseph F. Lingevitch \\ Naval Research Laboratory, Washington, D.C. 20375
}

\author{
Andrew J. Bernoff \\ Department of Engineering Science \& Applied Mathematics, Northwestern University, Evanston, Illinois \\ 60208
}

(Received 8 September 1995; accepted 24 April 1997)

\begin{abstract}
Using fully nonlinear simulations of the two-dimensional Navier-Stokes equations at large Reynolds number (Re), we bracket a threshold amplitude above which a perturbed Gaussian monopole will relax to a quasi-steady, rotating tripole, and below which will relax to an axisymmetric monopole. The resulting quasi-steady structures are robust to small perturbations. We propose a means of measuring the decay rate of disturbances to asymptotic vortical structures wherein streamlines and lines of constant vorticity correspond in some rotating or translating frame. These experiments support the hypothesis that small or moderate deviations from asymptotic structures decay through inviscid and viscous mixing. (C) 1997 American Institute of Physics.
\end{abstract} [S1070-6631(97)02708-6]

\section{INTRODUCTION}

Laboratory experiments and numerical simulations of two-dimensional, large Reynolds number (Re) flows reveal the existence of isolated, long-lived vortical structures such as monopoles, dipoles, and tripoles. ${ }^{1-3}$ In this article, we study the evolution and relaxation of an axisymmetric Gaussian monopole perturbed by a quadrupolar vorticity disturbance of weak-to-moderate amplitude. We find that for sufficiently small amplitude nonaxisymmetric distortions, the perturbed monopole relaxes to an axisymmetric state, but for larger initial distortions, the vortex relaxes to a quasi-steady rotating tripole (see Fig. 1). Furthermore, we find that these tripole structures are robust to small disturbances. These numerical results highlight the existence of monopole and tripole attractors at large Reynolds numbers, and we suggest shearing and diffusion may even enhance the rate at which the perturbed initial conditions approach these attractors. They also suggest the existence of a perturbation threshold which separates the monopole and tripole domains of attraction.

The decay rate of vortical structures toward an attractor is associated with the homogenization of disturbances through mixing. If disturbances to one structure are mixed more efficiently than for another structure, the former will relax much more quickly. In the experiments described in this paper, we increase the amplitude of the disturbance to a point where mixing no longer occurs across the entire monopole. When this happens, we find that disturbances are not homogenized in some regions. Rather, part of the disturbance persists with the original monopole, yielding a completely different stable attractor called a tripole.

The simultaneous shearing and diffusion of vorticity governs the intermediate timescale $\left(\mathrm{Re}^{1 / 3}\right)$ relaxation of perturbed monopoles and, we suspect, tripoles. Rhines and Young studied the shear-diffusion mechanism in their analysis of the mixing of a passive scalar in a region of closed streamlines. ${ }^{4}$ They showed that shear steepens concentration gradients across streamlines which are then smoothed by diffusion, homogenizing the concentration along streamlines on the $\mathrm{Pe}^{1 / 3}$ timescale, much faster than the Pe diffusive timescale (Pe is the Péclet number). Recently, we incorporated this mechanism into the linear stability theory for an axisymmetric monopole. ${ }^{5}$ This analysis shows that a linearly perturbed monopole relaxes to an axisymmetric state on the shear-diffusion timescale $\left(\operatorname{Re}^{1 / 3}\right)$. Unfortunately, measuring decay toward a quasi-steady, nonaxisymmetric structure, such as the tripoles observed in our experiments, is problematic because there is no direct way to measure the distance of an evolving state from the asymptotic state without an asymptotic solution. This contributes to our failure to differentiate between the $\mathrm{Re}$ timescale and the $\mathrm{Re}^{1 / 3}$ timescale. However, we do formulate a consistent measurement technique that can measure the decay to a certain point in the evolution of the nonaxisymmetric structure.

Tripoles have been observed in numerical and laboratory experiments of decaying turbulence and dipole-dipole collisions for some time., ${ }^{2,6,7-10}$ Our full nonlinear simulations of distorted axisymmetric monopoles suggest that sheardiffusion mixing governs the relaxation toward the tripole attractor for a moderate perturbation just as it would toward the monopole attractor for slight perturbations. In the case of the tripole, the positive portion of the quadrupolar disturbance is thoroughly mixed, while the negative portion persists to form a quasi-steady rotating tripole. The persistence of the negative portion of the perturbation is due to the creation of a separatrix of the streamlines in a frame rotating with the vortical structure by the perturbation vorticity. The effect of this distortion is to nullify the shearing of the negative vorticity inclusions by the background velocity field. Therefore, shear-diffusion mixing cannot occur between the negative inclusion and the rest of the structure. A similar inviscid experiment with an elliptical top-hat vorticity profile indicated rapid axisymmetrization in the sense described ear- 

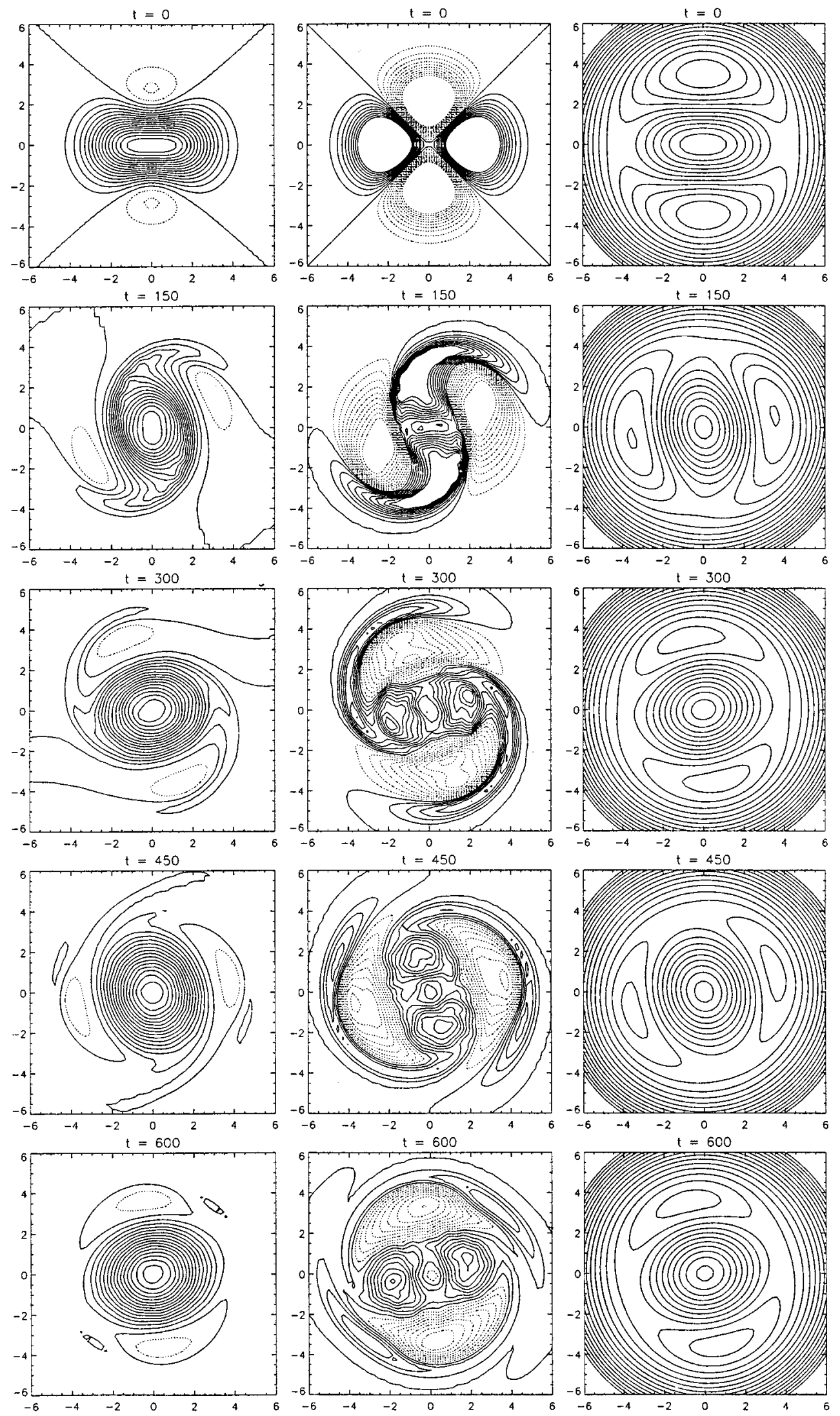

FIG. 1. A perturbed monopole relaxes toward a tripolar attractor if the perturbation $(\delta=0.25)$ is strong enough. The full vorticity field is on the left, the perturbation relative to the Lamb monopole base state is in the center, and the corotating stream function (see Sec. IV) is on the right. Time increases from top to bottom. The contours are remain constant throughout the series $\left(5.0 \times 10^{-2}\right.$ per division (left) and $1.25 \times 10^{-3}$ per division (middle). Dotted contours are negative.) The final structure persists for the remainder of the simulation. 
lier, but since there was no negative vorticity, there could be no local disruption of shear within the structure. ${ }^{11}$ The ramifications of this work are that nonaxisymmetric perturbations to a stable monopole may not decay back to the base state, as some investigators have assumed in both analytic and numerical work. ${ }^{12-14}$

\section{MIXING IN VORTICAL STRUCTURES}

Large Reynolds number flows exhibit the emergence and persistence of a variety of vortical structures. ${ }^{2,3}$ Quasi-steady vortices are those that maintain approximately the same vorticity distribution in some appropriate reference frame for many turnover times and evolve solely through viscous diffusion on a slow $(\mathrm{Re})$ timescale. Fundamental examples are monopoles (stationary), dipoles (translating), and tripoles (rotating). Viewed in their respective reference frames, quasi-steady vortices are characterized by the approximate alignment of their vorticity contours and streamlines: $\mathbf{u} \cdot \nabla \omega \approx 0$. It is known that mixing in regions of closed streamlines may greatly enhance the effectiveness of diffusion in homogenizing passive tracers along streamlines. ${ }^{4}$ Similarly, mixing has also been shown to enhance the decay of nonaxisymmetric disturbances to axisymmetric monopoles. ${ }^{5}$ In this section, we examine how mixing in regions of closed streamlines may effect the relaxation of a perturbed vortex to a stable quasi-steady state. First, we illustrate the mechanism of shear-diffusion mixing in the context of the large Reynolds number linear stability of an exact monopole solution to the Navier-Stokes equations, the Lamb monopole. Then, we speculate on how mixing applies more generally to vortices and formulate several numerical experiments to investigate the extent and limitations of mixing in vortices. In the next section, we discuss the results of our numerical simulations.

The large Reynolds number stability of the Lamb monopole illustrates how mixing enhances the decay of nonaxisymmetric disturbances to a axisymmetric monopole much quicker than the viscous timescale. Below, we formulate the large Reynolds number $(\operatorname{Re} \gg 1)$ monopole problem that we solve numerically in Sec. III. We are studying the evolution of a two-dimensional, incompressible flow which is described by the Navier-Stokes equations

$$
\partial_{t} \omega+\mathbf{u} \cdot \nabla \omega=\frac{1}{\operatorname{Re}} \nabla^{2} \omega,
$$

where $\omega$ is the total vorticity, $\mathbf{u}$ is the velocity field related to the vorticity through the Biot-Savart integral, and Re is the Reynolds number, which for our experiments is defined to be the total circulation divided by the viscosity. ${ }^{15}$ An exact, self-similar, axisymmetric solution of Eq. (1) is the Lamb monopole,

$$
\omega_{0}(\mathbf{x}, t)=\frac{1}{4 \pi(1+t / \operatorname{Re})} \exp \left[-\frac{|\mathbf{x}|^{2}}{4(1+t / \operatorname{Re})}\right] .
$$

This Gaussian vortex is exponentially localized and spreads radially on the viscous timescale $\left(\mathrm{Re}^{-1}\right)$ through viscous diffusion. The Lamb solution serves as a canonical example of an axisymmetric large Reynolds number monopole and is the basic state which we perturb with nonaxisymmetric distortions. In fact, all axisymmetric monopoles will relax to a Gaussian monopole as $t \rightarrow \infty .{ }^{16}$ However, while shear diffusion plays no role in axisymmetric vortex evolution, it may significantly affect the relaxation of nonaxisymmetric perturbations to the monopole. To examine the decay of a nonaxisymmetric perturbation to a Lamb monopole, we define the nonaxisymmetric enstrophy of the monopole:

$$
\begin{aligned}
& E_{n}=\int[\omega(\mathbf{x})-\langle\omega(|\mathbf{x}|)\rangle]^{2} d \mathbf{x}, \\
& \langle\omega(|\mathbf{x}|)\rangle=\frac{1}{2 \pi} \int_{0}^{2 \pi} \omega(\mathbf{x}) d \theta .
\end{aligned}
$$

This is a natural quantification for axisymmetric structures because it represents the $L_{2}$ norm of the nonaxisymmetric portion of the vorticity field. Using this measurement, the linear stability theory for a Lamb monopole was examined in a previous paper. ${ }^{5}$ Below, we briefly review the main results of the stability theory as a motivation for our study of finite amplitude distortions to Gaussian monopoles. To study the relaxation of more general perturbed quasi-steady vortices, the nonaxisymmetric enstrophy is not the appropriate measurement for quantifying the deviation of the vorticity from its quasi-steady state. In Sec. IV, we formulate a more general technique that measures decay relative to corotating vortical structures.

To study perturbed monopoles, it is useful to incorporate the Lamb solution into Eq. (1) and to obtain an equation for the perturbation vorticity. The total vorticity is written as a sum of the Lamb solution, $\omega_{0}$, and a perturbation, $\omega^{\prime}$. The evolution of $\omega^{\prime}$ is governed by

$$
\partial_{t} \omega^{\prime}+\mathbf{u}_{0} \cdot \nabla \omega^{\prime}+\mathbf{u}^{\prime} \cdot \nabla \omega_{0}+\mathbf{u}^{\prime} \cdot \nabla \omega^{\prime}=\frac{1}{\operatorname{Re}} \nabla^{2} \omega^{\prime},
$$

where $\mathbf{u}_{0}, \mathbf{u}^{\prime}$ are the velocity fields induced by the Lamb monopole and perturbation vorticity, respectively. The linear stability equation follows from Eq. (5) by neglecting the quadratic nonlinear term $\left(\mathbf{u}^{\prime} \cdot \nabla \omega^{\prime}\right)$.

Shear-diffusion mixing of vorticity perturbations in the Lamb monopole is qualitatively similar to the mixing of passive scalars in shear flows, first studied by Rhines and Young. ${ }^{4}$ A passive scalar obeys a similar advection diffusion equation [i.e., Eq. (5) linearized and neglecting the $\mathbf{u}^{\prime} \cdot \nabla \omega_{0}$ term]. A nonaxisymmetric initial distribution of vorticity (or passive scalar) is sheared by differential rotation in the monopole. Diffusion between the sheared layers homogenizes the vorticity (or passive scalar) along streamlines on the shear-diffusion timescale $\mathrm{Re}^{1 / 3}$ (or $\mathrm{Pe}^{1 / 3}$, where $\mathrm{Pe}$ is the Péclet number). In linear theory, a general vorticity perturbation is decomposed into three independent components: axisymmetric, translational, and the portion which is orthogonal to the axisymmetric and translational components. The axisymmetric portion of the disturbance radially diffuses on the Re timescale, analogously to the base state. The translational piece represents a small displacement of the vorticity centroid of the monopole and is conserved. The remaining portion of the perturbation decays on the shear-diffusion $\left(\operatorname{Re}^{1 / 3}\right)$ timescale. Note that the decay timescale, $\left(\operatorname{Re}^{1 / 3}\right)$, is 
asymptotically (i.e., as $\mathrm{Re} \rightarrow \infty$ ) faster than the viscous spreading of the monopole $(\mathrm{Re})$. A linearly perturbed Gaussian vortex will therefore become axisymmetric on the sheardiffusion timescale, much quicker than the Lamb monopole diffuses. The result of the Lamb monopole linear stability analysis is that the shear-diffusion mechanism attenuates the residual portion (i.e., nonaxisymmetric, nontranslational component) of the vorticity perturbation on the $\mathrm{Re}^{1 / 3}$ timescale at a rate proportional to $n^{2}$, where $n$ is the azimuthal wave number of the disturbance. ${ }^{5}$

The shear-diffusion mixing mechanism fails in the regions where shear vanishes such as translation or solid body rotation. In this case, there is no differential rotation between neighboring streamlines. The Kirchoff elliptical vortex patches fall into this category. Another limitation of sheardiffusion mixing is that the perturbation vorticity may qualitatively alter the stream function so that shearing due to the base state is insufficient to mix the vorticity. In the next section, we see an example of this as the perturbation amplitude to a monopole is increased beyond a threshold, and the vortex relaxes to a quasi-steady tripole rather than a monopole.

To determine the extent and limitations of sheardiffusion mixing in perturbed monopoles, we numerically simulate finite amplitude distortions to the Lamb monopole. The linear stability theory discussed above is applicable to large Reynolds numbers; numerical solutions of the linearized equation show good agreement with large Reynolds number $\left(\operatorname{Re}=10^{4}\right)$ asymptotic scaling. ${ }^{5}$ The numerical simulations discussed in this paper have Reynolds number between $\operatorname{Re}=10^{3}$ and $\operatorname{Re}=10^{4}$. Our numerical simulations are for finite amplitude quadrupolar perturbations to a Lamb monopole. The initial vorticity disturbance is given by

$$
\omega^{\prime}(\mathbf{x}, 0)=\frac{\delta}{4 \pi}|\mathbf{x}|^{2} \exp \left(-\frac{|\mathbf{x}|^{2}}{4}\right) \cos (2 \theta),
$$

where $\theta$ is the argument of $\mathbf{x}$. We note that although we refer to the perturbed vortex as a distorted monopole, it is also accurate to say that the initial state is a tripole with strength proportional to $\delta$. When $\delta$ is small, the nonlinear term, $\mathbf{u}^{\prime} \cdot \nabla \omega^{\prime}$ is of higher order in Eq. (5), and the evolution of the perturbed monopole is dominated by linear effects and decays on the $\mathrm{Re}^{1 / 3}$ timescale as discussed above. we chose to study the $n=2$ azimuthal fourier mode because this perturbation is the slowest decaying fourier mode, with the exception of portions of the $m=1$ mode that are orthogonal to a translation of $\omega_{0}$.

In this section, we have described how mixing may modify the inviscid dynamics of perturbed quasi-steady vortex on the $\mathrm{Re}^{1 / 3}$ timescale due to the shear-diffusion mechanism. This means for large Reynolds number flows, viscosity may enhance the equilibration to a quasi-steady state much faster than the Re timescale. We expect shear to create small scale structures which are annihilated by viscosity. Also, we have discussed how shear is essential for this rapid mixing process so that one might expect only viscous diffusion to act in regions where there is little or no shear.

\section{THE MONOPOLE RELAXATION EXPERIMENTS}

We simulated the evolution and morphology of the perturbed monopole using the corrected core spreading vortex method (CCSVM). ${ }^{17}$ However, we implemented two different versions of it for different regimes. One version directly solves Eq. (1); the other solves Eq. (5). When $\delta$ is large and the perturbation is comparable to the unperturbed Lamb monopole, we directly simulated the full vorticity field. The disadvantage to a direct simulation is that the full field, $\omega_{0}$ $+\omega^{\prime}$, is calculated, ignoring the fact an exact solution for $\omega_{0}$ is known. Thus numerical errors will accumulate in both fields. The principle advantage is that the direct calculation has no operator splitting for reasons that will be discussed in greater detail in the following paragraphs. In contrast to the direct method, approximating the perturbation equation (5) and using the exact solution for $\omega_{0}$ allows numerical errors to grow only in $\omega^{\prime}$. A similar perturbative technique has proven successful in boundary layer calculations. ${ }^{18}$ Since $\omega^{\prime}$ is $\mathscr{Q}(\delta)$ and $\omega_{0}$ is $\mathscr{Q}(1)$, this method is effective for small values of $\delta$. However, an additional spatial error is introduced in the perturbative method because the split step scheme is used.

The Lagrangian vortex formulation is a natural one for a detailed study of high Reynolds number monopole relaxation for several reasons. First, the moving basis functions are free to convect anywhere in the domain when approximating the expulsed spiral arms of vorticity. Second, the boundary conditions for an isolated monopole are naturally satisfied. Spectral calculations would simulate the relaxation of a periodic array of monopoles. Finally, the corrected core spreading method incorporates true viscous diffusion rather than artificial dissipation such as the more controversial hyperviscosity $\left(\nabla^{4}\right){ }^{19}$

As discussed in Sec. II, shear and diffusion play a crucial role in monopole relaxation for infinitesimal perturbations. Clearly, the $\mathrm{Re}^{1 / 3}$ relaxation time is much faster than the $\mathrm{Re}$ diffusion timescale, but more importantly, the fast relaxation scaling depends strongly on the type of diffusion. In this case, the appropriate type of diffusion would be viscous diffusion $\nu \nabla^{2} \omega$. Intuitively, simulations using hyperviscosity would result in $\mathrm{Re}^{1 / 5}$ decay rather than the physically correct $\mathrm{Re}^{1 / 3}$ for linearized simulations. ${ }^{4}$ Specifically, if one studies a simple model problem of a passive scalar in a simple shear flow, the exact solution will decay on the $\mathrm{Re}^{1 / 5}$ timescale for hyperviscosity. More generally, hyperviscosity will balance the shear terms in the Navier-Stokes equations at the same order $\left(\operatorname{Re}^{1 / 5}\right)$. Other forms of dissipation such as those used in contour surgery techniques have not been quantified in the literature, and we do not pursue its application in this article. Although we are studying high Reynolds number flows where the shear-diffusion mechanism is applicable, it is crucial that the numerical dissipation is accurate at the correct order.

\section{A. Exact initial conditions}

Initially, the vorticity field is approximated as a linear combination of Gaussian basis functions: 


$$
\omega(\mathbf{x})=\sum_{i=1}^{N} \frac{\gamma_{i}}{4 \pi \sigma_{i}^{2}} \exp \left(-\frac{\left|\mathbf{x}-\mathbf{x}_{i}\right|^{2}}{4 \sigma_{i}^{2}}\right) .
$$

The positions of the basis functions are arranged on a regular grid to approximate the initial vorticity field $\omega_{0}(\mathbf{x}, 0)$ $+\omega^{\prime}(\mathbf{x}, 0)$. The most direct way to assign circulations to the basis functions is

$$
\gamma_{i}=f\left(\mathbf{x}_{i}\right) \Delta \mathbf{x}_{i}=\left[\omega_{0}\left(\mathbf{x}_{i}, 0\right)+\omega^{\prime}\left(\mathbf{x}_{i}, 0\right)\right] \Delta \mathbf{x}_{i},
$$

where $\Delta \mathbf{x}_{i}$ is the area surrounding mesh node $\mathbf{x}_{i}$. Assuming that the Gaussians overlap sufficiently, the error of this approximation is $\mathscr{Q}(\sigma)$ if $\sigma_{i}=\sigma$ for all $i$. This error is not an interpolation error due to having a finite number of elements but rather a regularization error. As one refines the grid (in the limit as $\Delta \mathbf{x}_{i}$ grows small and the number of basis functions grows large), the initial conditions of the computation would converge to

$$
\iint f\left(\mathbf{x}^{\prime}\right) \frac{1}{4 \pi \sigma^{2}} \exp \left(-\frac{\left|\mathbf{x}-\mathbf{x}^{\prime}\right|^{2}}{4 \sigma^{2}}\right) d \mathbf{x}^{\prime} .
$$

However, there is another choice of $f$, different from Eq. (8), that cancels the regularization error.

The procedure, referred to as "deregularization," takes advantage of the fact that certain functions can be expressed as regularizations of other functions. For example, the simplest example would be that a Gaussian function of width $\sigma_{0}$ can be expressed as a regularization of a Gaussian function with width $\sigma_{1}$ regularized with basis functions of width $\sqrt{\sigma_{0}^{2}-\sigma_{1}^{2}}$. It is also possible to deregularize the function $\omega_{0}(\mathbf{x}, 0)+\omega^{\prime}(\mathbf{x}, 0)$. In particular, by choosing

$$
\begin{aligned}
f(\mathbf{x})= & \frac{1}{4 \pi\left(1-\sigma^{2}\right)}\left[1+\frac{\delta}{\left(1-\sigma^{2}\right)^{2}}|\mathbf{x}|^{2} \cos (2 \theta)\right] \\
& \times \exp \left[-\frac{|\mathbf{x}|^{2}}{4\left(1-\sigma^{2}\right)}\right] .
\end{aligned}
$$

This $f$ will yield the exact initial conditions subject only to interpolation errors.

\section{B. The vorticity computation}

The vorticity is approximated by a linear combination of Gaussian basis functions that convect with the velocity determined from the base state and the basis functions via the Biot-Savart law. The viscous term is approximated by allowing the basis functions to spread:

$$
\begin{gathered}
\dot{\mathbf{x}}_{i}=\left[\begin{array}{cc}
0 & -1 \\
1 & 0
\end{array}\right] \sum_{j=1}^{N} \frac{\gamma_{i}}{2 \pi} \frac{\mathbf{x}_{i}-\mathbf{x}_{j}}{\left|\mathbf{x}_{i}-\mathbf{x}_{j}\right|^{2}}\left[1-\exp \left(-\frac{\left|\mathbf{x}_{i}-\mathbf{x}_{j}\right|^{2}}{4 \sigma_{i}^{2}}\right)\right], \\
\dot{\sigma}_{i}^{2}=\nu
\end{gathered}
$$

Systematically, single wide elements split into thinner elements to maintain numerical consistency in a process called adaptive spatial refinement, effectively diffusing vorticity across streamlines. Thus computational elements are continually spreading, splitting into configurations of thinner elements, and then spreading again. To control problem size, groups of overlapping elements are periodically merged when this action induces a controllably small error. ${ }^{14}$

To calculate a solution to Eq. (5), it is necessary to split the operator by alternatively solving Eq. (1) for $\omega^{\prime}$ rather than $\omega$, and then solving

$$
\partial_{t} \omega^{\prime}=-\left(\mathbf{u}^{\prime} \cdot \nabla\right) \omega_{0}
$$

for one time step. Computationally, one integrates each equation for one full time step. In this case, Eq. (13) is approximated by adding computational elements on a regular grid. Naively, each element should have a circulation

$$
\gamma_{k}=f\left(\mathbf{x}_{k}\right) \Delta \mathbf{x}_{k} \Delta t=-\left[\mathbf{u}^{\prime}\left(\mathbf{x}_{k}\right) \cdot \nabla\right] \omega_{0}\left(\mathbf{x}_{k}\right) \Delta \mathbf{x}_{k} \Delta t,
$$

where $\mathbf{x}_{k}$ is the location on a grid node, and $d \mathbf{x}_{k}$ is the area of the grid. As discussed in the previous subsection, each element is Gaussian of finite thickness, and the intuitive choice of $f$ in Eq. (14) will result in a finite regularization error. However, since the forcing function is not known a priori, as is the case with the initial conditions, an exact deregularization is not possible.

This problem is solved with an "approximate deregularization" applied locally. A local deregularization is where the function $-\left[\mathbf{u}^{\prime}\left(\mathbf{x}_{k}\right) \cdot \nabla\right] \omega_{0}\left(\mathbf{x}_{k}\right)$ is locally expanded as

$$
\begin{aligned}
& {\left[\mathbf{u}^{\prime}\left(\mathbf{x}_{k}\right) \cdot\left(\mathbf{x}-\mathbf{x}_{k}\right)+\mathcal{Q}\left(\left|\mathbf{x}-\mathbf{x}_{k}\right|^{2}\right)\right] \frac{1}{8 \pi(1+\nu t)^{2}}} \\
& \quad \times \exp \left[-\frac{\left|\mathbf{x}-\mathbf{x}_{k}\right|^{2}}{4(1+\nu t)}\right],
\end{aligned}
$$

taking advantage of the fact that the base state is known. This function can be deregularized similar to the initial conditions:

$$
f(\mathbf{x})=\frac{\mathbf{u}^{\prime}(\mathbf{x}) \cdot \mathbf{x}}{8 \pi\left(1+\nu t-\sigma^{2}\right)^{2}} \exp \left[-\frac{|\mathbf{x}|^{2}}{4\left(1+\nu t-\sigma^{2}\right)}\right] .
$$

The major difference is that the formula assumes that $\mathbf{u}^{\prime}$ does not vary much over the numerical length scale $\sigma$.

\section{Verification of nonlinear calculations}

We have addressed three distinct concerns that encompass our numerical experiments. First, both numerical methods used to calculate our solutions are self-consistent. That is, as numerical parameters were refined, our solutions converged to a solution, resolving all appropriate length scales. Since CCSVM has been shown to be uniformly convergent to Eq. (1), this was simply a matter of conducting a refinement study. Second, for small $\delta$, the perturbative solution agrees with detailed $k=2$ mode linear calculations. Third, the two methods are consistent with one another for large $\delta$. Unfortunately, after several turnover times, it is not clear from our measurements whether or not we are resolving the small length scales necessary to observe shear-diffusion decay. Whether it is numerical errors in the calculation or systematic errors in our ability to determine the asymptotic tripole state (see Sec. IV) is not clear. Regardless, these three detailed studies verify our numerical results, most particularly our decay measurements, during the beginning and intermediate stages of relaxation. 
Decay of Nonaxisymmetric Enstrophy

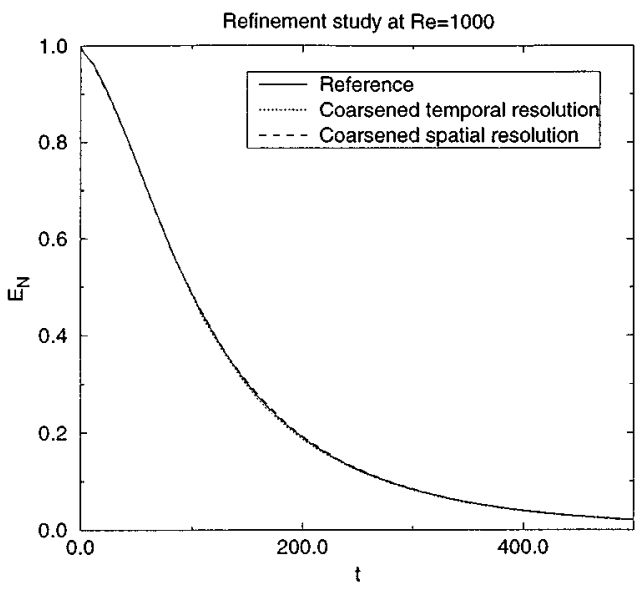

FIG. 2. Nonaxisymmetric enstrophy decay for $\delta=0.25$ while spatial resolution $l$ and the viscous timestep, $\Delta t$, are varied by a factor of 0.2 and 0.5 , respectively. Even with if the spatial resolution is $20 \%$ coarser or the temporal resolution is $50 \%$ coarser, there is little noticable difference in nonaxisymmetric enstrophy decay.

To make sure that our finite amplitude nonlinear simulations were resolved and self-consistent, we performed standard refinement studies. The system of ordinary differential equations described by Eqs. (11) and (12) are solved using a second-order Adams-Bashforth scheme. The time step was reduced until uniform variations in the vorticity distribution dropped below 0.01 at $T=250$. The Lagrangian technique described above has two spatial numerical parameters. The first parameter is the maximum length scale, $l$, in the calculation so that $\sigma_{i}<l$ for all computational elements. The second parameter is nondimensional and governs the accuracy of the spatial refinement when $\sigma_{i}=l$. However, this parameter can also be expressed as a timescale called the viscous time step, $\Delta t$. The viscous time step is the period of time a computational element travels between refinement processes. Thus a resolved calculation should have a relatively small variation in nonaxisymmetric enstrophy decay as the viscous time step is decreased. In Fig. 2, one can see that refinement of the spatial parameters does not substantially alter the enstrophy decay. Thus assured that we had resolved the spatial and temporal evolution, we calculate the decay of the perturbed monopole over a range of Reynolds numbers and amplitudes.

The numerical parameters of the experiment must be sufficiently fine to resolve all the important structures in the decaying monopole. The most obvious qualitative comparison can be drawn from linear results. In this case, simulating Eq. (5) for a small value of $\delta$ should yield similar results to the linear equations. Since the linear equations describe the evolution of a single Fourier mode, they are solved spectrally on a very fine grid and are fully resolved. In Fig. 3, a comparison is made between a linear calculation and low amplitude $(\delta=0.02)$ nonlinear calculation. Visually, one can see that they are quite similar, although some nonlinear effects are still present in the low amplitude simulation. In particular, the symmetry between positive and negative vorticity is
Radial distribution of nonaxisymmetric vorticity.
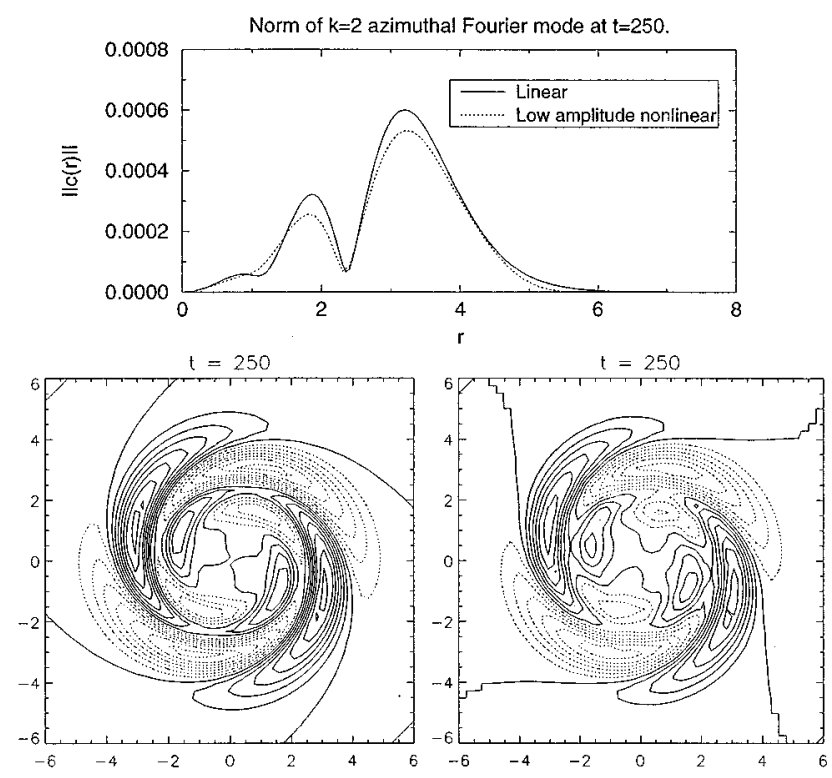

FIG. 3. Top: The radial distribution of nonaxisymmetric vorticity at $t$ $=250$ for the linear and low amplitude $(\delta=0.02)$ nonlinear simulation. $\|c(r)\|$ is the Fourier amplitude as a function of $r$. Below: The vorticity distributions for the linear (left) and nonlinear (right) simulations show very close correspondence. The contour interval is $1.6 \times 10^{-4}$.

broken. This is evident near the origin where zero contour bifurcates in the linear simulation but does not in the nonlinear simulation. In the nonlinear calculation, the positive lobes have migrated slightly inward and the negative lobes are displaced slightly outward. However, the nonlinear simulation does preserve the reflection symmetry of the positive and negative vorticities individually. Most significantly, we can compute the $k=2$ azimuthal mode amplitude from the nonlinear simulation and compare it to its linear calculation. Again, the agreement is quite sound. The deviations between the curves can be attributed to splitting errors (both time discretization and spatial regularization) from the perturbative method.
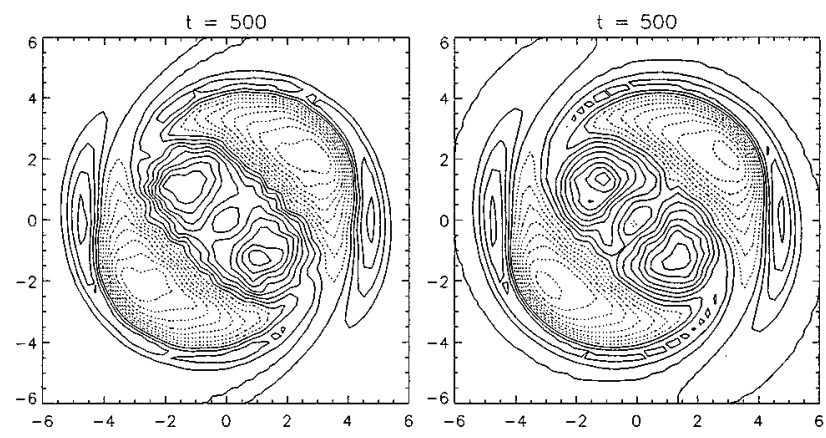

FIG. 4. Comparison of perturbed vorticity calculations (left) to direct vorticity calculations (right). Even for large values of $\delta$ and therefore large amplitude forcing functions, the simulation of Eq. (5) captures the monopole evolution almost as well as the direct simulation. If the $e$ is the error between these two fields, then $\|e\|_{\infty} /\left\|\omega_{0}\right\|_{\infty}=0.056$ and $\|e\|_{2} /\left\|\omega_{0}\right\|_{2}=0.044$. 


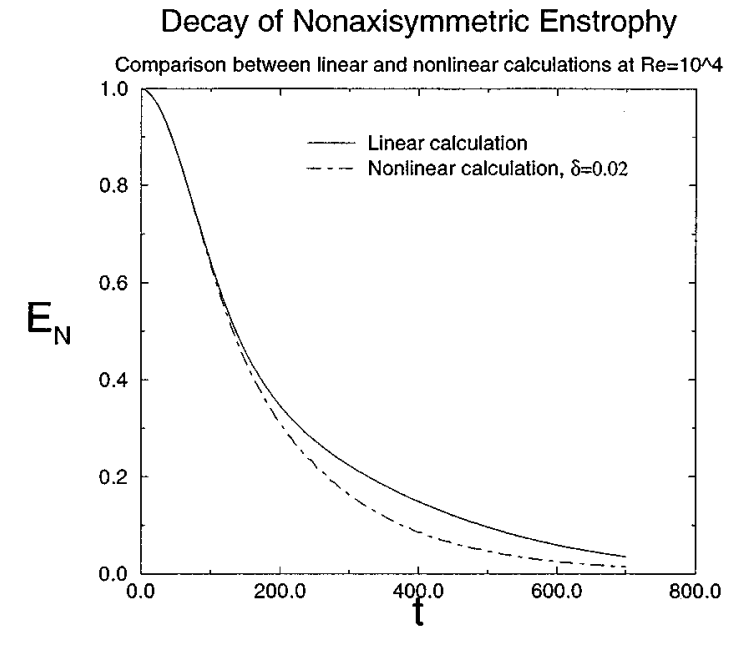

FIG. 5. A comparison between the nonaxisymmetric enstrophy of linear and nonlinear monopole relaxation simulations. The linear simulation uses a linear spectral code capable to resolving shear-diffusion spatial scales. CCSVM is a fully nonlinear code, but cannot resolve the very fine spatial scales necessary for shear-diffusion mixing as well as the linear spectral simulation.

To determine the limitations of the perturbative technique due to splitting and regularizations error, we calculated the same flow with both perturbative and direct methods at a large value of $\delta=0.25$. At large values of $\delta$, one would expect the performance of the direct method to improve over that of the perturbative method entirely due to splitting errors. In Fig. 4, the slow exponential growth of the splitting errors is evident but manageably small relative to the total nonaxisymmetric enstrophy decay.

\section{ANALYSIS OF RELAXATION TOWARD THE MONOPOLE AND TRIPOLE ATTRACTORS}

Our relaxation experiments examine monopole relaxation as $\delta$ and Re vary. We examined monopole relaxation for $\delta=0.02,0.1,0.25$ at $\mathrm{Re}=10^{4}$ to see the effect of the nonlinearity on the base state. Also, we examined $R e=10^{3}$, $2.5 \times 10^{3}, 5.0 \times 10^{3}, 7.5 \times 10^{3}$, and $10^{4}$ when $\delta=0.25$ to observe how viscous diffusion affects perturbed monopole evolution. This section describes and discusses our two main conclusions. First, perturbed Lamb monopoles do not necessarily return to a state of radial symmetry. For small $\delta(0.02$ and 0.1 ), the positive and negative aspects of the disturbance mix together and negate one another, leaving the original Lamb monopole essentially intact. However, for large enough $\delta(0.25)$, the initial tripolar structure undergoes a period of reorganization that leaves the negative inclusions intact. The second conclusion is that the nonaxisymmetric tripole resulting from a moderate disturbance is robust and only slowly decays through viscous diffusion. As we decrease the Reynolds number, we decrease the lifetime of the tripole because viscosity erodes the negative inclusions.

Our greatest frustration is that we do not observe rapid shear-diffusion mixing in the tripolar attractor. There are two possible reasons why this is the case. The first is that numerical errors within our nonlinear simulation hinder our ability to measure an effect which is itself quite small. That is, the finest scales of our simulation may not be quite fine enough to capture shear diffusion mixing. This is evident in Fig. 5 wherein we compare the nonaxisymmetric enstrophy (discussed further in the next section) for linear decay with a low amplitude nonlinear simulation. However, it is clear that the nonlinear simulations are capturing a monotonic decay in close agreement to the linear simulation. The second problem is that we lack an analytic or numerical solution to the tripole attractor. Indeed, as we shall see later, the tripole is not even steadily rotating, and there are some small radial oscillations as well. Thus, measuring the decay relative to a quasi-steady structure is somewhat problematic although we do propose a technique that captures some of these effects.

\section{A. Measurements of decaying structures in corotating frames}

When studying decay toward an attractor, it is necessary to first know the structure of the attractor. In the case of the linear stability of the Lamb monopole, there is an exact solution to the stable structure. In the case of the quasi-steady tripole, we lack an exact solution. Even moving into a frame that is rotating with the tripole, there are slight radial oscillations in the negative inclusions. In fact, we shall see later that the tripole does not rotate steadily. Rather, the rotation rate varies slightly with time. Thus measuring the rate of decay of this quasi-steady object is problematic. In this section, we propose a new technique for measuring decay rates of structures like the tripole.

Although nonaxisymmetric enstrophy is an appropriate quantity for measuring relaxation to axisymmetric attractors, it would not be appropriate for general vortical structures because the attractor might not have axisymmetric streamlines. To measure the relaxation in a quasi-steady rotating object where the streamlines of the attractor are not known, we move to the frame rotating with the structure. Melander, McWilliams, and Zabusky used a similar method of analysis to study their top-hat elliptical vortices. ${ }^{11}$ If one knows the angular velocity, $\Omega$, for the attractor, one can determine the corotating stream function and its associated velocity field:

$$
\begin{aligned}
& -\omega(\mathbf{x}, t)+2 \Omega=\nabla^{2} \psi_{\mathrm{rot}}, \\
& \mathbf{u}_{\mathrm{rot}}=\left[\begin{array}{c}
-\partial_{y} \\
\partial_{x}
\end{array}\right] \psi_{\mathrm{rot}} .
\end{aligned}
$$

Convection across corotating streamlines associated with mixing would then be measured through $\mathbf{u}_{\mathrm{rot}} \cdot \nabla \omega$, the convective term of the Navier-Stokes equations. Thus a natural relaxation measurement is the convective orthogonality,

$$
F=\int_{0}^{2 \pi} \int_{0}^{\infty} \frac{\left(\mathbf{u}_{\mathrm{rot}} \cdot \nabla \omega\right)^{2}}{\left\|\mathbf{u}_{\mathrm{rot}}^{2}\right\|} r d r d \theta .
$$

Normalization of the velocity field is essential because we only wish to measure vorticity transport across streamlines and $\psi_{\text {rot }}$ grows quadratically in $r$. If the vortex structure is steadily rotating or even if $\Omega$ changes slowly with time (i.e., locally steadily rotating), one would expect $F \rightarrow 0$ as $t \rightarrow \infty$ physically. For calculations, there would be some floor value at which one observes numerical errors. 
Since these measurements rely on knowing the base state's angular velocity $\Omega$, we developed a consistent computational technique for determining a local rotation rate for the vortex structure. Since an exact solution to the tripole base state is not known, we computed the rotation rate locally using second moments. The zeroth and first moments of the tripole structure are conserved both physically and numerically and so cannot provide any relevant information about the angular orientation of a structure. However, the individual second moments evolve with time and, from this information, we can determine the rotation rate.

Using second moments, we determined the angular velocity of the evolving tripole with centered differences. Suppose we measure the following quantities:

$$
\begin{aligned}
& m_{x x}(t)=\iint x^{2} \omega(r, \theta, t) r d r d \theta, \\
& m_{x y}(t)=\iint x y \omega(r, \theta, t) r d r d \theta, \\
& m_{y y}(t)=\iint y^{2} \omega(r, \theta, t) r d r d \theta .
\end{aligned}
$$

There are three unique second moments corresponding to the three coefficients of a two-dimensional quadratic form. If the structure is rotating steadily with angular velocity $\Omega$, then

$$
\begin{gathered}
{\left[\begin{array}{ccc}
m_{x x}(t) & 2 m_{x y}(t) & m_{y y}(t) \\
m_{x y}(t) & m_{y y}(t)-m_{x x}(t) & -m_{x y}(t) \\
m_{y y}(t) & -2 m_{x y}(t) & m_{x x}(t)
\end{array}\right]} \\
\times\left[\begin{array}{c}
\cos ^{2}(\Omega \Delta t) \\
\sin (\Omega \Delta t) \cos (\Omega \Delta t) \\
\sin ^{2}(\Omega \Delta t)
\end{array}\right]=\left[\begin{array}{l}
m_{x x}(t+\Delta t) \\
m_{x y}(t+\Delta t) \\
m_{y y}(t+\Delta t)
\end{array}\right] .
\end{gathered}
$$

Thus to uniquely determine the three quantities $\left(\cos ^{2}, \sin ^{2}\right.$, and $\cos \sin$ ) of interest based on the three moments, it is necessary to solve this three by three system. This linear system is easily inverted yielding a local value of $\Omega$. As a diagnostic, we calculated $\sin ^{2}(\Omega \Delta t)+\cos ^{2}(\Omega \Delta t)$ as the perturbed monopole evolved and found that this residual deviated from unity by less than $0.1 \%$ during the simulation. One subtlety in solving this problem is that it is best to compute

$$
\Omega=\tan ^{-1}\left[\frac{\sin (\Omega \Delta t) \cos (\Omega \Delta t)}{\cos ^{2}(\Omega \Delta t)}\right] /(\Delta t)
$$

rather than

$$
\Omega=\tan ^{-1}\left[\frac{\sin ^{2}(\Omega \Delta t)}{\sin (\Omega \Delta t) \cos (\Omega \Delta t)}\right] /
$$

because $\Delta t$ is small and the latter will induce large numerical errors.

\section{B. Quasi-steady tripoles and local negation of shear diffusion}

Our first observation in measuring $F$ is that the rotation rate of the tripolar attractor is not steady. In Fig. 6, one can see that even after the tripole relaxes to a quasi-steady structure, the rotation rate varies with time. Furthermore we observe subtle radial motion in the structure, as well further
Variations in Local Angular Velocity

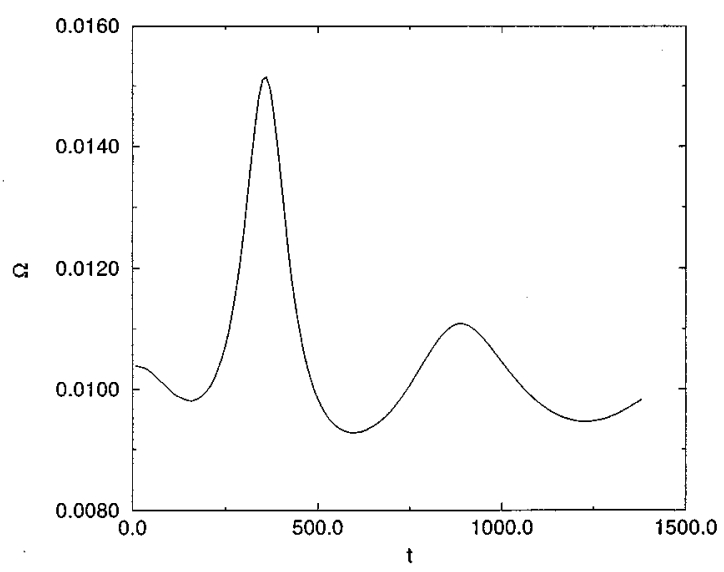

FIG. 6. Slow evolution of $\Omega$ for the tripolar attractor. Although only comprising a portion of the total angular velocity, significant variations in $\Omega$ are observed for $\delta=0.25$ and $\operatorname{Re}=10^{4}$. The angular velocity, $\Omega$, is determined using Eq. (21).

hindering measurements of relaxing toward the tripole attractor. This oscillation in the aspect of the structure may be connected to a similar oscillation in the axisymmetrization experiments Melander, McWilliams, and Zabusky measured with "diagnostic ellipses." 11 However, it is important to note that these previous experiments study the axisymmetrization of top-hat elliptical profiles which are more akin to Kirchoff vortices than more generic continuous distributions of vorticity where shear diffusion will be present. The second observation is that, while our simulations can capture the initial stages of the relaxation where the perturbation becomes sheared and stretched to fine scales, either radial redistribution in the tripole or numerical noise affects our measurements before shear-diffusion mixing can occur. Although we cannot isolate an asymptotic decay timescale, we can identify the strong nonlinear, inviscid mixing mechanism as we vary Re.

Nonlinear interactions manifest themselves in several different ways as the perturbation relaxes. The nonlinear interactions radially redistribute the axisymmetric mode so that
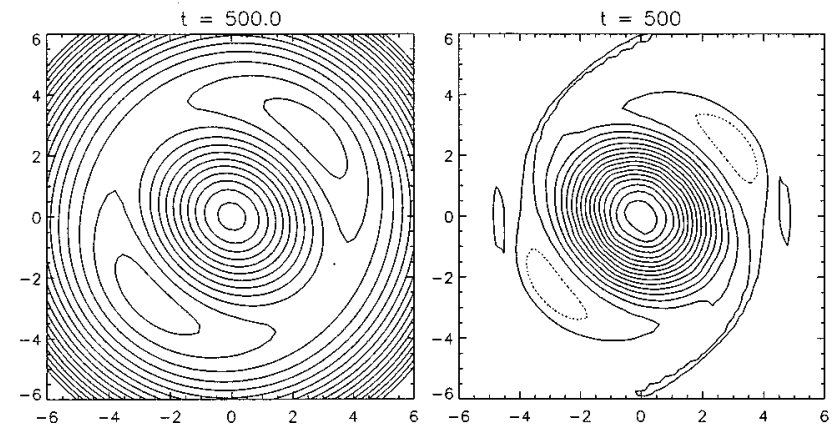

FIG. 7. The stream function, $\psi_{\text {rot }}$, (left) for $\delta=0.25$ for $\omega$ in a frame corotating with the tripole and the perturbed vorticity, $\omega^{\prime}$, (right) at $t=500$. The angular velocity of the tripole, $\Omega$, is observed to be $2 \pi / 620$. The vorticity contour interval is $5.0 \times 10^{-2}$. 

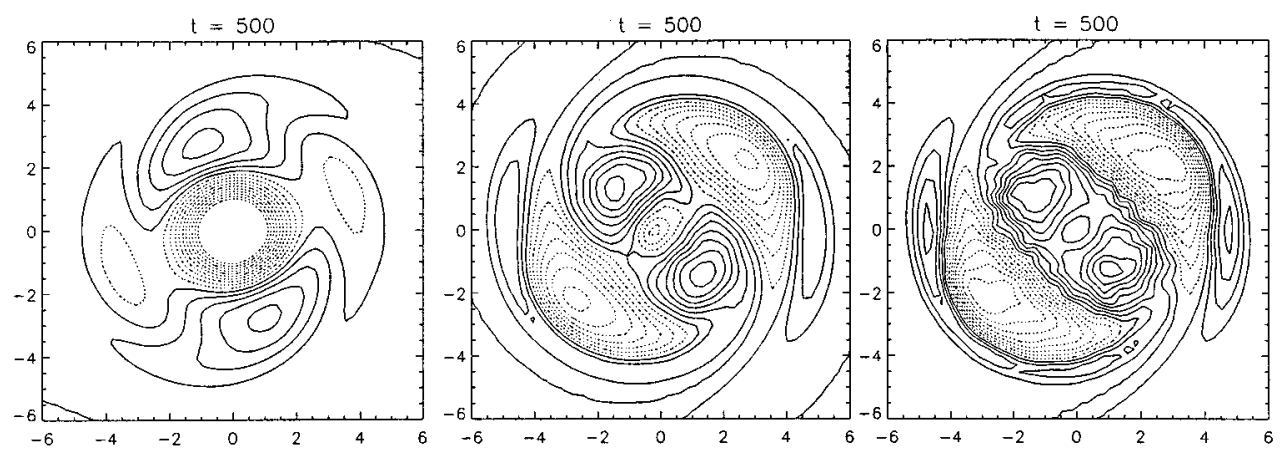

FIG. 8. Gradual erosion of tripole structure as the Reynolds number decreases: As one can see from these snapshots of moderately perturbed $(\delta=0.25)$ monopole disturbances, for a fixed time the structure gradually erodes as one increases the Reynolds number. From left to right, Re $=10^{3}, 5 \times 10^{3}$ and $10^{4}$. The vorticity contours are the same $\left(1.25 \times 10^{-3}\right.$ per division) throughout the sequence.

the symmetric part of the total vorticity field is no longer a Lamb monopole. This structure, once created, relaxes slowly on the Re timescale. Next, the negative vorticity inclusions can create a critical separatrix and closed streamlines, counteracting shear diffusion in certain regions of the flow corresponding to the negative vorticity inclusions (see Fig. 7). Thus it is impossible for the shear diffusion to eradicate the negative inclusions while stripping can occur outside this separatrix. We suspect that this mechanism is responsible for coherence of the tripole. In a sense, the fundamental problem reduces to studying the stability of simpler structures in shear flows. The stability of elliptical vortex structures in shear flows has been studied by Kida, and Moore, and Saffman. ${ }^{20,21}$ However, the instability of an elliptical form does not imply a lack of coherence of a more general structure. Similarly, Mariotti, Legras, and Dritschel have studied the stripping of vorticity by cooperative and adverse shearing as a critical mechanism in vortex erosion although they also found that viscous diffusion greatly enhances erosion. ${ }^{19}$

While nonlinear interactions would prevent sheardiffusion mixing between the negative inclusions and the rest

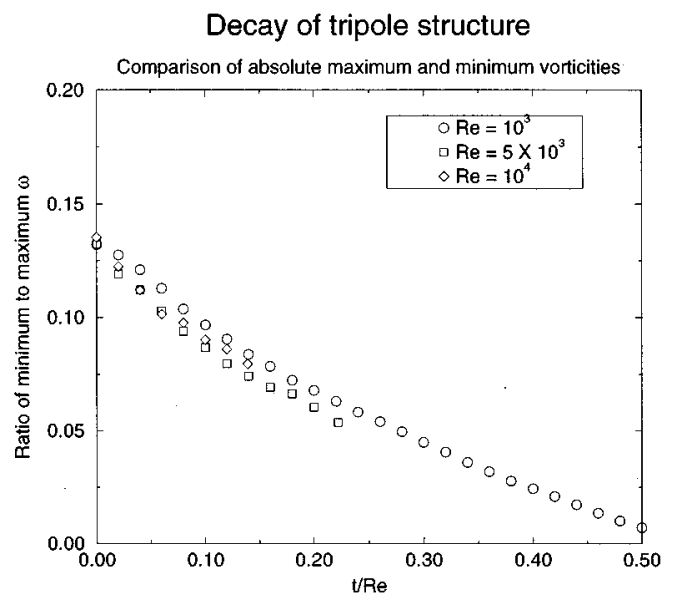

FIG. 9. Reynolds number timescale decay of the ratio between the maximum vorticity and minimum vorticity. While there is some initial reorganization, the asymptotic tripoles at various Reynolds numbers decay in a similar manner. of the tripole, the tripole is still robust, meaning that both physical and computational disturbances to the structure are attenuated by some mechanism. One possible explanation is the shear-diffusion mechanism.

Eventually, viscosity transports vorticity across closed streamlines. In particular, positive vorticity will mix into the negative inclusions. In Fig. 8, one can see the effects of erosion at different Reynolds numbers. Since the structure is quasi-steady and vorticity is diffusing solely across streamlines, we would expect to see the tripole relax on a slow Re timescale (see Fig. 9). There is some retardation at the lower Reynolds numbers because the negative vorticity has diffused more substantially into the monopole. At $\operatorname{Re}=10^{3}$, one can no longer observe negative contours by $t=600$.

It is clear that the Gaussian monopole is the asymptotic structure for these experiments as $t \rightarrow \infty$ because the negative inclusions are vanishing relative to the base state. Even if there were a persistent viscous tripole solution, one would expect it to be unstable to external disturbances as $t \rightarrow \infty$ because the negative inclusions would drop below any given value for large time. However, the existence of viscous tripole solutions for all time remains an open question.

\section{CONCLUSIONS}

In this paper, we describe in some generality how perturbed monopoles will evolve toward one of two distinct attractors. The monopole attractor is already well understood, but the tripole attractor remains elusive. While these tripoles are robust to moderate perturbations, it is not clear what the asymptotic structure is. While we speculate that the asymptotic structure would correspond to an alignment between vorticity contours and streamlines, there is no definitive evidence that this is the case. Finally, we propose a technique for measuring the decay of perturbations to the speculative asymptotic state.

The observation of this tripole attractor contradicts the natural assumption that concentrated regions of vorticity where the field is dominated by positive (or negative) vorticity will rapidly relax to an axisymmetric state. For instance, some investigators have assumed that vortex sheets will roll up and aggregate into an axisymmetric core region. ${ }^{13}$ While 
this may be true in many situations, these tripole experiments indicate that there are exceptions where these assumptions are not valid. Similarly, in vortex method computations, overlapping vortices are merged in special circumstances to decrease the computational complexity. ${ }^{12,14}$ These experiments indicate that this assumption is not correct in all circumstances.

\section{ACKNOWLEDGMENTS}

We wish to thank the Pittsburgh Supercomputer Center (DMS-940016P) for the use of their Cray C90. L.F.R. acknowledges support of the NSF Mathematical Sciences Postdoctoral Research Fellowship (DMS-9407660). J.F.L. and A.J.B. acknowledge support of NSF Grant No. CTS9206828. The authors would also like to thank the unnamed referees for their valuable comments and suggestions on this work.

${ }^{1} \mathrm{X}$. Carton and B. Legras, "The life-cycle of tripoles in two-dimensional incompressible flows,', J. Fluid Mech. 267, 53 (1994).

${ }^{2} \mathrm{~S}$. Gama and U. Frisch, "Simulations of two-dimensional turbulence on the connection machine,', Appl. Sci. Res. 51, 105 (1993).

${ }^{3}$ J. C. McWilliams, "The emergence of isolated coherent vortices in turbulent flow,', J. Fluid Mech. 146, 21 (1984).

${ }^{4} \mathrm{P}$. B. Rhines and W. R. Young, "How rapidly is a passive scalar mixed within closed streamlines,', J. Fluid Mech. 133, 133 (1983).

${ }^{5}$ A. J. Bernoff and J. F. Lingevitch, "Rapid relaxation of an axisymmetric vortex,', Phys. Fluids 6, 3717 (1994).

${ }^{6}$ J. B. Flor, W. S. S. Govers, G. J. F. Van Heijst, and R. Van Sluis, "Formation of a tripolar vortex in a stratefied fluid,' Appl. Sci. Res. 51, 405 (1993).

${ }^{7}$ M. Ikeda, "Instability and splitting of mesoscale rings using a two-layer quasi-geostrophic model on an $f$-plane,'” J. Phys. Ocean. 11, 987 (1981).

${ }^{8}$ G. Y. Morel and J. X. Carton, "Multipolar vortices in two-dimensional incompressible flows," J. Fluid Mech. 267, 23 (1994).

${ }^{9}$ L. M. Polvani and X. J. Carton, "The tripole: A new coherent vortex structure of incompressible two-dimensional flows,' Geophys. Astrophys. Fluid Dynamics 51, 87 (1990).

${ }^{10} \mathrm{M}$. Swenson, "Instability of equivalent-barotropic riders,', J. Phys. Ocean. 17, 492 (1987).

${ }^{11}$ M. V. Melander, J. C. McWilliams, and N. J. Zabusky, “Axisymmetrization and vorticity-gradient intensification of an isolated two-dimensional vortex through filamentation,' J. Fluid Mech. 178, 137 (1987).

${ }^{12}$ T. Y. Hou, J. Lowengrub, and M. J. Shelley, "Exact desingularization and local regriding for vortex methods,', in Lectures in Applied Mathematics: Vortex Methods and Vortex Dynamics, edited by C. Greengard (American Mathematical Society, New York, 1991), pp. 341-362.

${ }^{13}$ T. S. Lundgren, "Strained sprial vortex model for turbulent fine structure,', Phys. Fluids 25, 2193 (1982).

${ }^{14}$ L. F. Rossi, "Merging computational elements in Lagrangian simulations,' SIAM J. Sci. Comp. 18-4, 1 (1997).

${ }^{15}$ G. K. Batchelor, An Introduction to Fluid Dynamics (Cambridge University Press, New York, 1967).

${ }^{16}$ L. Ting and R. Klein, Viscous Vortical Flows, Vol. 374 of Lecture Notes in Physics (Springer-Verlag, New York, 1991).

${ }^{17}$ L. F. Rossi, "Resurrecting core spreading methods: A new scheme that is both deterministic and convergent," SIAM J. Sci. Comp. 17-2, 370 (1996).

${ }^{18}$ L. F. Rossi, "Vortex computations of wall jet flows," in The 1st Annual Forum on Vortex Methods for Engineering Applications, pp. 127-147 (1995).

${ }^{19}$ A. Mariotti, B. Legras, and D. G. Dritschel, "Vortex stripping and the erosion of coherent structures in two-dimensional flows," Phys. Fluids 6, 3954 (1994)

${ }^{20}$ S. Kida, "Motion of an elliptic vortex in a uniform shear flow," J. Phys. Soc. Jpn. 50, 3517 (1981)

${ }^{21}$ D. W. Moore and P. G. Saffman, "Structure of a line vortex in an imposed strain,' in Aircraft Wake Turbulence, edited by J. H. Olsen, A. Goldburg, and M. Rogers (Plenum, New York, 1971), pp. 339-354. 